\title{
Adaptor Protein Complexes 1 and 3 Are Essential for Generation of Synaptic Vesicles from Activity-Dependent Bulk Endosomes
}

\author{
Giselle Cheung and Michael A. Cousin \\ Centre for Integrative Physiology, University of Edinburgh, Edinburgh, Scotland, United Kingdom EH8 9XD
}

Activity-dependent bulk endocytosis is the dominant synaptic vesicle retrieval mode during high intensity stimulation in central nerve terminals. A key event in this endocytosis mode is the generation of new vesicles from bulk endosomes, which replenish the reserve vesicle pool. We have identified an essential requirement for both adaptor protein complexes 1 and 3 in this process by employing morphological and optical tracking of bulk endosome-derived synaptic vesicles in rat primary neuronal cultures. We show that brefeldin A inhibits synaptic vesicle generation from bulk endosomes and that both brefeldin A knockdown and shRNA knockdown of either adaptor protein 1 or 3 subunits inhibit reserve pool replenishment from bulk endosomes. Conversely, no plasma membrane function was found for adaptor protein 1 or 3 in either bulk endosome formation or clathrin-mediated endocytosis. Simultaneous knockdown of both adaptor proteins 1 and 3 indicated that they generated the same population of synaptic vesicles. Thus, adaptor protein complexes 1 and 3 play an essential dual role in generation of synaptic vesicles during activity-dependent bulk endocytosis.

\section{Introduction}

Neuronal activity stimulates the fusion of neurotransmittercontaining synaptic vesicles (SVs) with the nerve terminal plasma membrane. SVs that are available for exocytosis in central nerve terminals are commonly referred to as the recycling pool (Sudhof, 2000), which can be further subdivided into the readily releasable pool (RRP) and the reserve pool. The RRP contains SVs that are immediately available for fusion, whereas the reserve pool only contributes SVs during periods of intense neuronal activity (Sudhof, 2000).

Neuronal activity is also a key determinant in the triggering of specific SV retrieval modes after exocytosis. Clathrin-mediated endocytosis (CME) is the dominant SV retrieval mode during mild stimulation (Granseth et al., 2006; Zhu et al., 2009), whereas during increased activity activity-dependent bulk endocytosis (ADBE) dominates (Clayton et al., 2008). ADBE is a high capacity SV retrieval mode that forms endosomes directly from large invaginations of the nerve terminal plasma membrane (Clayton and Cousin, 2009a). SVs that are generated from bulk endosomes specifically repopulate the reserve pool (Richards et al., 2000; Cheung et al., 2010), suggesting a functional link between reserve

\footnotetext{
Received Dec. 19, 2011; revised Feb. 13, 2012; accepted March 3, 2012.

Author contributions: M.A.C. and G.C. designed research; G.C. performed research; G.C. analyzed data; M.A.C. and G.C. wrote the paper.

This work was supported by a grant from the Wellcome Trust (Ref. 084277). The anti-delta SA4 antibody was obtained from the Developmental Studies Hybridoma Bank developed under the auspices of the NICHD and maintained by The University of lowa, Department of Biology, lowa City, IA 52242. Dr. Alan Prescott and Mr. John James (both University of Dundee) are thanked for excellent technical assistance.

The authors declare no competing financial interests.

Correspondence should be addressed to Mike Cousin, Membrane Biology Group, Centre for Integrative Physiology, George Square, University of Edinburgh, EH8 9XD, Scotland, UK. E-mail: M.Cousin@ed.ac.uk.

DOI:10.1523/JNEUROSCI.6305-11.2012

Copyright $\odot 2012$ the authors $\quad 0270-6474 / 12 / 326014-10 \$ 15.00 / 0$
}

pool mobilization and ADBE triggering (Clayton and Cousin, 2009a; Shupliakov, 2009).

The formation of functional SVs from donor membrane requires the efficient sorting of protein cargo, a role performed at the plasma membrane by the adaptor protein (AP) 2 complex during CME (Royle and Lagnado, 2003). The molecules that mediate SV generation from bulk endosomes during ADBE are still unknown. This process is thought to be clathrin dependent (Heerssen et al., 2008; Kasprowicz et al., 2008), suggesting that AP complexes will also be required. Five different AP complexes have been identified (AP-1 to AP-5) that mediate vesicle formation at specific donor membranes (Boehm and Bonifacino, 2001; Robinson, 2004; Hirst et al., 2011). In both non-neuronal and neuroendocrine cells, endosomal vesicle budding is inhibited by brefeldin A (BFA), which inhibits the GTPase ADP-ribosylation factor 1 (ARF1) (Drake et al., 2000). ARF1 is essential for the recruitment of both AP-1 and AP-3 to membrane, implicating both AP-1 and AP-3 in endosomal vesicle generation (Faundez et al., 1998; Pagano et al., 2004; Newell-Litwa et al., 2007).

A potential role for either AP-1 or AP-3 in SV generation from bulk endosomes is supported by previous studies. Both AP complexes are enriched in central nerve terminals (Glyvuk et al., 2010; Newell-Litwa et al., 2010) and are present on SVs (Takamori et al., 2006). Furthermore, SV endocytosis is sensitive to BFA only during intense activity in neuronal culture (Voglmaier et al., 2006; Kim and Ryan, 2009). Finally, mice lacking the $\sigma$ subunit of AP-1B display SV recycling defects, a large increase in endosome numbers, and a delay in recycling pool replenishment after strong stimulation (Glyvuk et al., 2010).

We directly examined the role of AP- 1 and AP-3 in SV generation from bulk endosomes using recently established morphological and optical assays that specifically track SVs derived from this ADBE- 
A

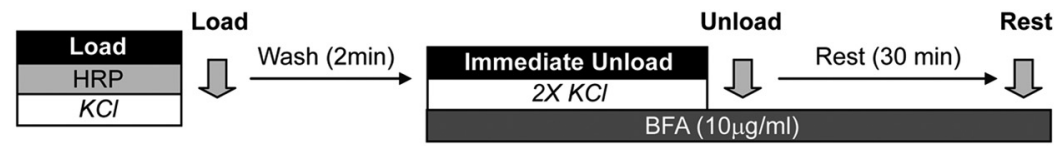

B

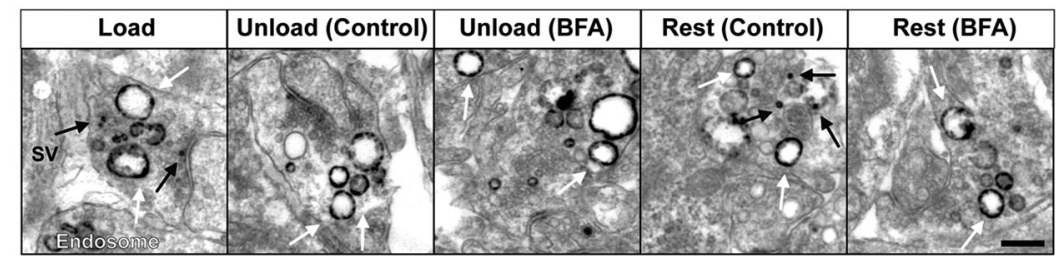

C

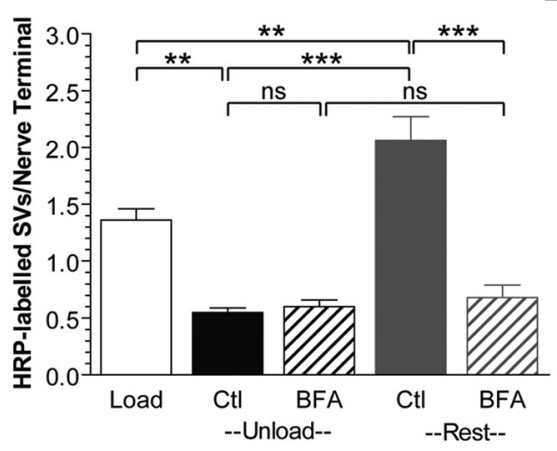

E

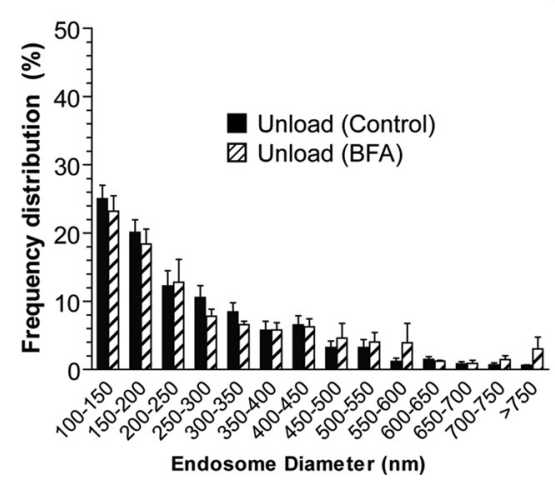

D

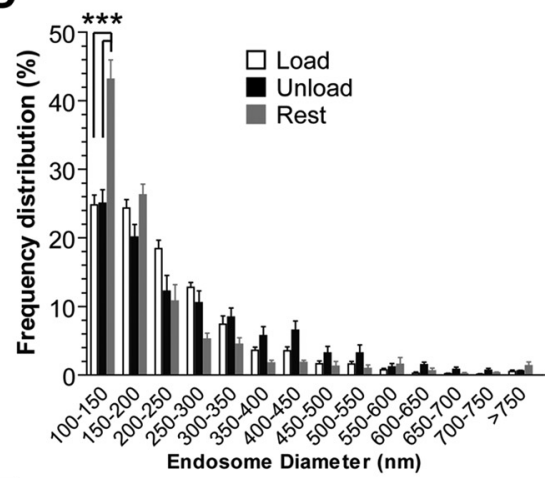

$\mathbf{F}$

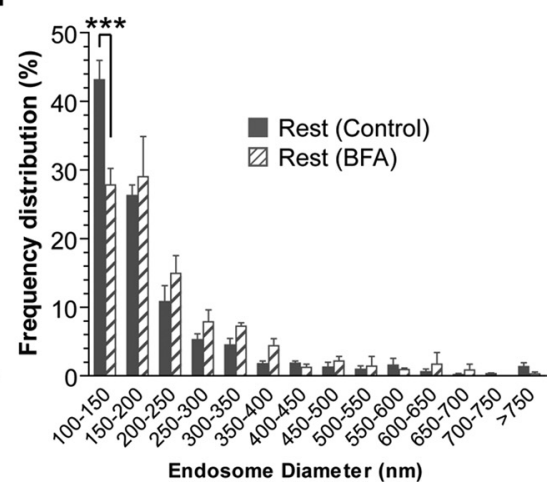

Figure 1. Brefeldin A arrests SV budding from bulk endosomes. $A$, Cultures were loaded with HRP ( $10 \mathrm{mg} / \mathrm{ml})$ in the presence of $50 \mathrm{~mm} \mathrm{KCl}$ for $2 \mathrm{~min}$. HRP was washed away immediately, and neurons were stimulated to release all available SVs using two sequential $30 \mathrm{~s} \mathrm{KCl}(50 \mathrm{~mm})$ stimuli. Cultures were then left to rest for $30 \mathrm{~min}$. Brefeldin A (10 $\mu \mathrm{g} / \mathrm{ml})$ was added to the cultures during the $\mathrm{KCl}$ unload and rest period. Cells were fixed either immediately after HRP loading (Load), immediately after $\mathrm{KCl}$ unloading (Unload), or after the 30 min rest period (Rest) as indicated by arrows. $\boldsymbol{B}$, Representative electron micrographs are shown for the treatments described above. Black arrows indicate HRP-labeled SVs, and white arrows indicate HRP-labeled endosomes (scale bar, $500 \mathrm{~nm}$ ). C, Bar graph displays the mean number of HRP-labeled SVs per nerve terminal for the indicated fixation points. For comparison, the mean number of HRP-labeled endosomes were: Load, $4.0 \pm 0.6$; Unload-Control, $5.7 \pm 0.7$; Unload$\mathrm{BFA}, 5.6 \pm 0.3$; Rest-Control, $4.4 \pm 0.5$; Rest-BFA, $3.7 \pm 0.6$ ( $n$ is number of nerve terminals [N is number of independent experiments]: Load, $n=551[N=6]$; Unload-Control, $n=227[N=4]$; Unload-BFA, $n=123[N=3]$; Rest-Control, $n=232$ $[N=4]$; Rest-BFA, $n=95[N=3]$; all $\pm S E M,{ }^{* * *} p<0.001,{ }^{* *} p<0.01$, one-way ANOVA using $\left.N\right)$. $\boldsymbol{D}-\boldsymbol{F}$, Frequency distribution of endosome diameter during Load (white bars), Unload (black bars), and Rest (gray bars) periods under control conditions (D), and either Unload $(\boldsymbol{E})$ or Rest $(\boldsymbol{F})$ in the presence or absence of BFA (hatched bars) ( $n$ is number of HRP endosomes, with total endosome number in parentheses: Load, $n=1980$ (3678); Unload-Control, $n=1245$ (2320); Unload-BFA, $n=1980701$ (1447); RestControl, $n=781$ (1336); Rest-BFA, $n=339$ (617); all \pm SEM, ${ }^{* * *} p<0.001$, two-way ANOVA).

dependent compartment. We uncovered an essential requirement for both AP-1 and AP-3, highlighting a molecular locus in a key SV endocytosis mode triggered during high intensity stimulation.

\section{Materials and Methods}

FM1-43, FM2-10, penicillin/streptomycin, phosphate-buffered salts, fetal calf serum, Minimal Essential Medium, and Alexa Fluor 568 goat antimouse IgG antibody were purchased from Invitrogen. Glutaraldehyde and osmium tetroxide were from Agar Scientific. ProFection mammalian calcium phosphate transfection system was obtained from Promega. Anti-AP- $1 \gamma$ was purchased from BD Biosciences. Anti-AP-3 $\delta$ antibody was from Developmental Studies Hybridoma Bank. All other reagents were from Sigma. shRNAs targeting AP- $1 \gamma$ or AP- $3 \delta$ subunits were designed using the pSUPER vector system (pSUPER neo-GFP, OligoEngine) with the following oligonucleotides: Oligo AP-1a, CA AACGCATTGGCTATTTA;OligoAP-1b, GGAA TGCTATTCTGTATGA; Oligo AP-3a, ACAAAG TGTTCCTCAAGTA; Oligo AP-3b, CTATCATC CTGGAGAATCA. The pSUPER vector was engineered to express mCerulean as described previously (Cheung et al., 2010).

Primary cultures, transfections and immunofluorescence studies of cerebellar granule neurons. Preparation, transfections, and immunofluorescence studies of rat cerebellar granule neurons (prepared from 7-d-old pups of either sex) were carried out according to previously described protocols (Tan et al., 2003; Clayton et al., 2009). Neurons were used between 8 and $10 \mathrm{~d}$ in vitro for all experiments. In some experiments, cultures were transfected using a calcium phosphate precipitation protocol between 5 and $7 \mathrm{~d}$ in vitro and used after $72 \mathrm{~h}$. AP- $1 \gamma$ or AP-3 $\delta$ expression was separately monitored by immunofluorescence intensity. For quantification, the fluorescence intensity in transfected neurons was expressed as a percentage of the averaged expression in untransfected neurons in the same field of view. At least three independent experiments were performed, with five fields of view being assessed for each experiment.

\section{Fluorescence imaging of SV turnover}

Fluorescence imaging of SV turnover using FM dyes was performed as described previously (Cheung et al., 2010). Briefly, cultures were repolarized for $10 \mathrm{~min}$ in incubation medium [in mM: $170 \mathrm{NaCl}, 3.5 \mathrm{KCl}, 0.4 \mathrm{KH}_{2} \mathrm{PO}_{4}, 20$ TES ( $N$-tris [hydroxyl-methyl]-methyl-2-aminoethanesulfonicacid), $5 \mathrm{NaHCO}_{3}, 5$ glucose, $1.2 \mathrm{Na}_{2} \mathrm{SO}_{4}$, $1.2 \mathrm{MgCl}_{2}$, and $1.3 \mathrm{CaCl}_{2}$; at $\mathrm{pH}$ 7.4]. Cultures were then mounted in an imaging chamber (RC21BRFS, Warner Instruments). Invaginating membrane was loaded with either FM1-43 (10 $\mu \mathrm{M})$ or FM2-10 $(100 \mu \mathrm{M})$ by evoking SV turnover using electrical field stimulation delivered by platinum wires embedded in the imaging chamber ( 800 action potentials, $100 \mathrm{~mA}, 1 \mathrm{~ms}$ pulse width). After washout of excess FM dye, the dye was unloaded using a series of action potential trains. For FM1-43 experiments, cultures were immediately stimulated (Immediate Unload) with sequential trains of action potentials to first unload the RRP $(30 \mathrm{~Hz}, 2 \mathrm{~s})$ and then the reserve pool (three trains of $40 \mathrm{~Hz}, 10$ s). After a $30 \mathrm{~min}$ rest period, an identical unloading protocol was repeated (Second Unload). This protocol allows quantification of newly generated SVs that replenish the RRP and reserve pool. The average fluorescence drop for each unloading step was expressed as a percentage of the total SV recycling pool (RRP plus reserve pool) of the Immediate Unload, allowing comparison across multiple experiments. For transfected nerve terminals, this was further normalized to the equivalent drop in untransfected nerve terminals within the same field of view. 
S2/S1 experiments were performed using FM2-10 in which cultures underwent two rounds of FM dye loading and unloading, separated by a $20 \mathrm{~min}$ rest period. BFA $(10 \mu \mathrm{g} / \mathrm{ml})$ was present before and during the S2 unloading step. The total S2 fluorescence drop $(\Delta \mathrm{S} 2)$ was expressed as a ratio of the total $\mathrm{S} 1$ fluorescence drop $(\Delta \mathrm{S} 1)$ for the same nerve terminals. In all cases, at least three independent experiments were performed with 90 nerve terminals from each untransfected culture being assessed. In experiments using cultures transfected with shRNA, 10-30 transfected and untransfected nerve terminals in the same field of view were examined. Only nerve terminals displaying stimulation-dependent dye loading and unloading were selected for analysis. The kinetics of dye unloading were analyzed by normalizing the fluorescence unload between 1 and 0 and calculating the time taken for $50 \%$ of the dye to be released $\left(t_{1 / 2}\right)$.

Labeling of endocytic pathways by horseradish peroxidase. Cultures were fixed and processed for electron microscopy as described previously (Cheung et al., 2010). Briefly, cultures were placed in incubation medium for $10 \mathrm{~min}$ and then stimulated for $2 \mathrm{~min}$ with $50 \mathrm{~mm} \mathrm{KCl}$ in the presence of $10 \mathrm{mg} / \mathrm{ml}$ horseradish peroxidase (HRP). After washout of HRP, cultures were immediately stimulated with two consecutive $30 \mathrm{~s}$ applications of $50 \mathrm{~mm} \mathrm{KCl}$. Cultures were then left to rest for $30 \mathrm{~min}$. Cultures were incubated with BFA $(10 \mu \mathrm{g} / \mathrm{ml})$ during the unload and rest periods. Cultures were fixed in 2\% glutaraldehyde in phosphate buffered saline at one of the three fixation time points, either directly after HRP loading, after unloading, or after rest. After washing with $100 \mathrm{~mm}$ Tris, pH 7.4, cultures were exposed to $0.1 \%$ diaminobenzidine and $0.2 \% \mathrm{H}_{2} \mathrm{O}_{2}$ in 100 mM Tris until color developed. Cultures were then washed with $100 \mathrm{~mm}$ Tris, stained with $1 \%$ osmium tetroxide for $30 \mathrm{~min}$, and then dehydrated using ethanol series and polypropylene oxide and embedded using Durcupan. Samples were sectioned, mounted on grids, and viewed using a FEI Tecnai 12 transmission electron microscope. Nerve terminals were included in the analysis providing they contained small SVs, regardless of whether they contained HRP. Intracellular structures that were $<100 \mathrm{~nm}$ in diameter were arbitrarily designated to be SVs, whereas larger structures were considered endosomes. The average endosome diameter was obtained by taking the average of the longest and shortest diameters of individual endosomes using ImageJ (National Institutes of Health).

Fluorescence imaging of dextran uptake. Uptake of tetramethylrhodaminedextran $(40 \mathrm{kDa})$ was monitored as described previously (Clayton et al., 2009). Briefly, transfected cultures were placed in incubation medium for $10 \mathrm{~min}$ and then stimulated with a train of 800 action potentials $(80 \mathrm{~Hz})$ in the presence of $50 \mu \mathrm{M}$ tetramethylrhodamine-dextran. Noninternalized dextran was then washed away by perfusion for $2 \mathrm{~min}$. The number of dextran puncta per $100 \mu \mathrm{m}$ of transfected neurite was determined in each field of view. At least three independent experiments were performed, with at least six fields of view being assessed for each experiment.

Image acquisition, processing, and analysis. In all experiments, fluorescence signals were visualized using a Zeiss Axio Observer A1 epifluorescence microscope. Transfected neurons expressing mCerulean were identified using 430 $\mathrm{nm}$ excitation, whereas FM dye loading and unloading was monitored at 500 $\mathrm{nm}$ excitation (in all cases, emission $>535 \mathrm{~nm}$ ). Immunostaining and dextran uptake was visualized using $550 \mathrm{~nm}$ excitation and a bandpass (575-640 $\mathrm{nm})$ emission filter. Experiments using untransfected cultures were performed using a $20 \times$ air objective, while experiments with transfected cultures were performed using a $40 \times$ oil objective (both from Zeiss). All images were acquired using a Zeiss AxioCam CCD camera controlled by a Zeiss AxioVision Release software. Time-lapse FM images were acquired at $4 \mathrm{~s}$ intervals. All immunofluorescence and dextran uptake images were captured as z-stacks and processed as follows. Maximum intensity projections of stacks were generated using an ImageJ $\mathrm{Z}$ Project function. Background fluorescence was subtracted using the Rolling Ball BackgroundSubtraction plugin(http://rsbweb.nih.gov/ij/plugins/rolling-ball. $\mathrm{html}$ ). Total fluorescence intensity of immunostaining was measured from ROIs of identical size over cell bodies. Dextran puncta on transfected neurites were highlighted using the Colocalization Finder plugin (http://rsb.info.nih.gov/ij/plugins/colocalization-finder.html) and counted. The length of transfected neurite in each field of view was measured using the Simple Neurite Tracer plugin (http://pacific.mpi-cbg.de/wiki/index.php/
Simple_Neurite_Tracer). All calculations and statistical analyses were performed using Microsoft Excel and GraphPad Prism software.

\section{Results}

\section{Brefeldin A arrests SV budding from bulk endosomes}

$\mathrm{ADBE}$ is the dominant SV retrieval mode during intense neuronal activity in central nerve terminals (Clayton et al., 2008); however, the molecular mechanism responsible for generation of SVs from bulk endosomes is unknown. Recent genetic studies in model systems have suggested a requirement for clathrin in this process (Heerssen et al., 2008; Kasprowicz et al., 2008). To determine whether the endosomal adaptors AP-1 or AP-3 were required, we disrupted their function using the ARF1 inhibitor BFA in primary neuronal cultures. To ensure that we observed SV generation specifically from bulk endosomes, the following experimental protocol was performed. Both CME and ADBE were triggered using a maximal depolarizing stimulus $(50 \mathrm{mM} \mathrm{KCl})$ in the presence of the fluid phase marker HRP (Fig. 1A). SVs and bulk endosomes that were generated by CME and ADBE, respectively, are visible as HRP-labeled structures (Fig. 1B). HRPlabeled SVs were then depleted by two sequential stimuli of 50 $\mathrm{mm} \mathrm{KCl} \mathrm{(Fig.} 1 B, C$ ). Cultures were then left to rest for $30 \mathrm{~min}$, allowing the generation of new HRP-labeled SVs from bulk endosomes (Fig. 1A). This protocol thus allows tracking of HRPlabeled SVs specifically generated from bulk endosomes during a defined time window.

The immediate unload of HRP-labeled SVs resulted in over a $60 \%$ depletion of HRP-labeled SVs in control cultures (Fig. 1C). After the 30 min rest period a large $(>3.5$-fold) increase in HRPlabeled SVs was then observed (Fig. 1C), indicating that SVs had been generated from bulk endosomes. When HRP-labeled endosomes in control cultures were examined in more detail, there was no change in their diameter during stimulation (Load, $278 \pm 5 \mathrm{~nm}$; Unload, $295 \pm 8 \mathrm{~nm}$ ). However, after the $30 \mathrm{~min}$ rest period there was a significantly larger population of smaller endosomes, indicating again that SV budding had occurred (Fig. 1D). The mean HRPendosome diameter was also significantly smaller when compared to HRP-labeled endosomes either before or after the immediate unload stimulus ( $229 \pm 8 \mathrm{~nm}, p<0.001$, one-way ANOVA).

To determine whether ARF1 recruitment of either AP-1 or AP-3 were required for SV generation specifically from bulk endosomes, cultures were incubated with BFA $(10 \mu \mathrm{g} / \mathrm{ml})$ only after HRP exposure (and endocytosis) was complete (Fig. 1A). Therefore, this protocol ensures that bulk endosome generation is unaffected by the presence of BFA. BFA did not affect the depletion of HRP-labeled SVs (Fig. 1C) or the mean diameter of endosomes during the immediate unload (Control, $295 \pm 8 \mathrm{~nm}$; BFA, $290 \pm$ $7 \mathrm{~nm}$; Fig. $1 E$ ). In contrast, BFA abolished the generation of new HRP-labeled SVs during the 30 min rest period (Fig. 1C). This inhibition was accompanied by a significant reduction in the population of small HRP-labeled endosomes (mean diameter, $262 \pm 11 \mathrm{~nm}$; Fig. 1 F). Thus, BFA arrests: (1) generation of HRP-labeled SVs after endocytosis; and (2) a decrease in the diameter of HRP-labeled endosomes. Both lines of evidence indicate a role for the ARF1-dependent recruitment of either AP-1 and/or AP-3 in SV generation from bulk endosomes.

\section{Replenishment of the reserve pool via bulk endosomes is BFA sensitive}

Bulk endosome-derived SVs selectively replenish the reserve pool of SVs in nerve terminals (Richards et al., 2000; Cheung et al., 2010). Thus, the replenishment of the reserve pool is an independent indicator of SV production from bulk endosomes. To con- 

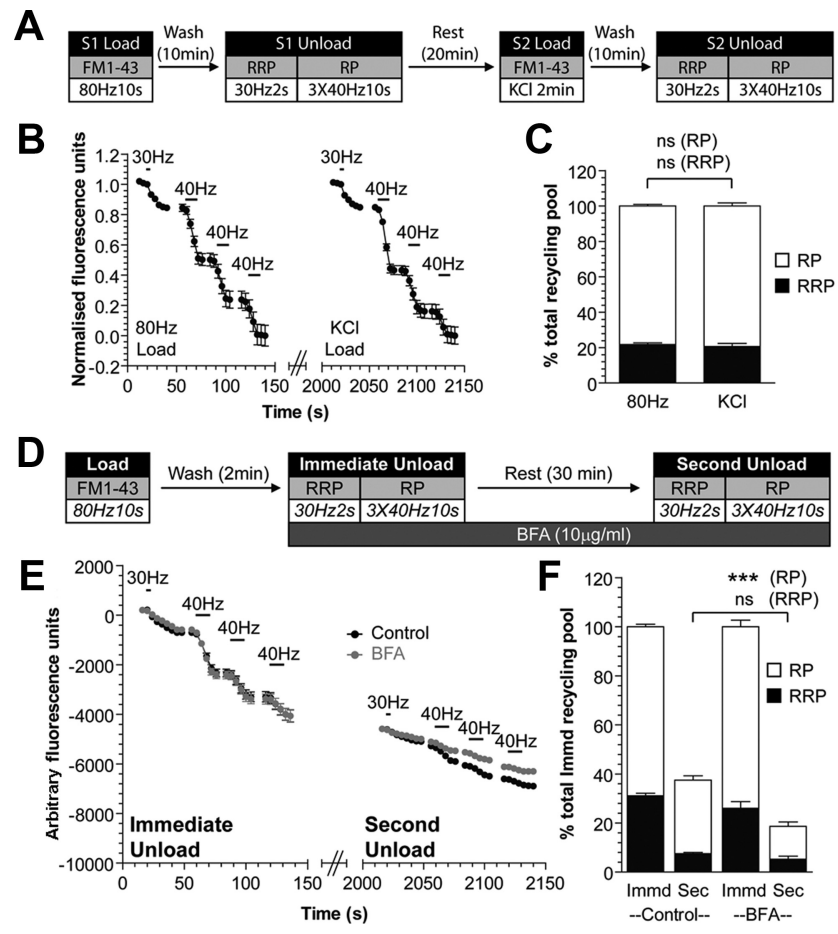

Figure 2. Brefeldin A inhibits SV reserve pool replenishment via bulk endosomes. $\boldsymbol{A}$, Cultures were subjected to an $\mathrm{S} 2 / \mathrm{S} 1$ protocol where they were loaded with FM1-43 (10 $\mu \mathrm{M})$ using a train of 800 action potentials $(80 \mathrm{~Hz})$ at $\mathrm{S1}$ and $\mathrm{KCl}(50 \mathrm{~mm}$ for $2 \mathrm{~min}$ ) at $\mathrm{S} 2$. Dye was washed away immediately and unloaded with $30 \mathrm{~Hz}, 2 \mathrm{~s}$ (RRP) and three trains of $40 \mathrm{~Hz}, 10 \mathrm{~s}$ (reserve pool, RP) sequentially in each instance. $\boldsymbol{B}$, Representative trace of dye unloading during both $\mathrm{S} 1$ and $\mathrm{S} 2$ unloads is shown. Both Immediate Unload and Second Unload traces are normalized to their respective recycling pool. C, Bar graphs display mean values of the size of the RRP and RP of each unload normalized to the total recycling pool; all $n=3 \pm$ SEM, one-way ANOVA. D, Cultures were loaded with FM1-43 (10 $\mu \mathrm{m})$ using a train of 800 action potentials $(80 \mathrm{~Hz})$. Dye was washed away immediately after stimulation and after $2 \mathrm{~min}$ the $\operatorname{RRP}(30 \mathrm{~Hz} 2 \mathrm{~s})$ and reserve pool ( $\mathrm{RP}$, three trains of $40 \mathrm{~Hz}, 10 \mathrm{~s}$ ) were sequentially unloaded (Immediate Unload). The same unloading stimuli were delivered after a 30 min rest period (Second Unload). Brefeldin A (10 $\mu \mathrm{g} / \mathrm{ml}$ ) was exposed to cultures before the Immediate Unload and was present thereafter. $\boldsymbol{E}$, Representative traces displaying the unloading of dye during both immediate and second unloads (black, control; gray, BFA). Traces are presented in arbitrary fluorescence units. Bars indicate the period of stimulation. $\boldsymbol{F}$, Bar graphs display mean values of the size of both the RRP and RP during both unloads, with the second unload normalized to total immediate recycling pool. All experiments, $n=3 \pm$ SEM, ${ }^{* * *} p<0.001$, one-way ANOVA.

A

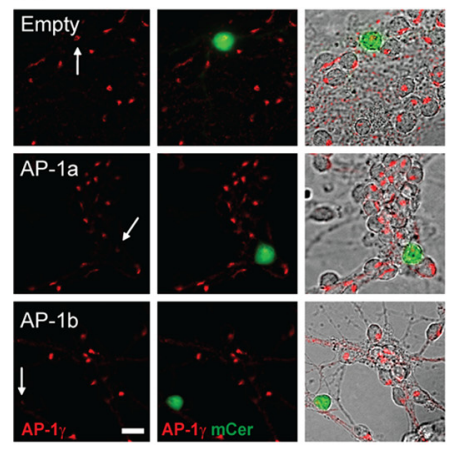

B

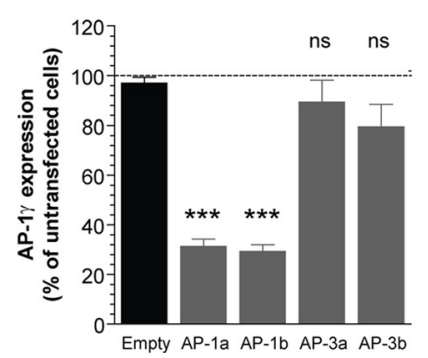

firm that BFA also inhibits the replenishment of the reserve SV pool, we performed an experiment in which we loaded cultures with the fluorescent dye FM1-43 using a train of 800 action potentials $(80 \mathrm{~Hz})$. This stimulation protocol triggers both $\mathrm{CME}$ and ADBE pathways, allowing FM1-43 to label both CMEderived SVs and ADBE-derived bulk endosomes (Clayton et al., 2008) and results in an identical pattern of dye loading to that observed using elevated $\mathrm{KCl}$ (Fig. $2 A-C$ ). Immediately after the loading of dye, FM1-43-loaded SVs were depleted by sequentially unloading the RRP ( 60 action potentials at $30 \mathrm{~Hz}$ ) and then the reserve pool (three 400 action potential trains at $40 \mathrm{~Hz}$ ) (Cheung et al., 2010). After a rest period of $30 \mathrm{~min}$ to allow SV generation from bulk endosomes, cultures were challenged again with identical unloading stimuli (Fig. 2D). Almost all released fluorescence originated from the reserve pool (percentage of second unload: RRP, $20.0 \pm 1.5$; Reserve, $80.0 \pm 4.75$; Fig. $2 E, F)$, confirming that the reserve pool is replenished by bulk endosome-derived SVs (Cheung et al., 2010).

To determine whether ARF1 is required for reserve SV pool replenishment, BFA was applied after washout of FM1-43 (Fig. $2 D)$. BFA had no effect on the fusion of either the RRP or the reserve pool during the immediate unloading challenge (Fig. $2 E, F)$. After the $30 \mathrm{~min}$ rest period, however, replenishment of the total recycling pool was significantly reduced (percentage of immediate unload recycling pool: Control, $37 \pm 2 \%$; BFA, $19 \pm$ $3 \% ; p<0.01$, Student's $t$ test), suggesting that replenishment of either or both of the RRP and reserve pool was retarded. When this was determined, a specific decrease in the replenishment of the reserve pool was observed in BFA-treated cultures (Fig. $2 E, F)$. Importantly the small replenishment of the RRP was unaltered (Fig. 2E,F). Thus, the inhibition of SV generation from bulk endosomes by BFA translates into a decreased replenishment of the reserve pool by these SVs.

To confirm that the observed effects of BFA were not a direct effect on SV exocytosis, we performed an S2/S1 experiment where the same nerve terminals undergo two identical cycles of dye loading and unloading. To ensure that the assay readout was not confounded by bulk endosome-dependent effects, we used the dye FM2-10, which does not label ADBE (Clayton et al., 2008; Clayton and Cousin, 2008). FM2-10 was loaded at both S1 and S2 with 800 action potentials $(80 \mathrm{~Hz})$ and unloaded $(\Delta \mathrm{S} 1$ and $\Delta \mathrm{S} 2)$ using three trains of 400 action potentials $(40 \mathrm{~Hz})$. Preincubation with BFA for

Figure 3. shRNAs targeting AP-1 $\gamma$ or AP-3 $\delta$ subunit specifically decreased their expression. $\boldsymbol{A}, \boldsymbol{C}$, Cultures were transfected with shRNA vectors that were either empty or expressing oligonucleotides against AP-1 $\gamma$ (AP-1a, AP-1b) or AP-3 $\delta$ (AP-3a, AP-3b). After $72 \mathrm{~h}$, the expression of AP-1 $\gamma$ or AP-3 $\delta$ was quantified using immunofluorescence. Representative images are shown for either AP-1 $\gamma(\boldsymbol{A})$ or AP-3 $\delta(\boldsymbol{C})$. Left panels show either AP-1 $\gamma(\boldsymbol{A})$ or AP-3 $\delta(\boldsymbol{C})$ immunostaining (red). Middle panels show transfected neurons ( $\mathrm{green}$ ). Right panels show a merged image with the corresponding bright-field image. White arrows indicate AP-1 $\gamma(\boldsymbol{A})$ or AP-3 $\delta(\boldsymbol{C})$ expression in transfected cells (scale bars, $10 \mu \mathrm{m}) . \boldsymbol{B}, \boldsymbol{D}$, Bar graphs display mean intensity of either AP-1 $\gamma(\boldsymbol{B})$ or $\operatorname{AP}-3 \delta(D)$ immunofluorescence in transfected neurons normalized to untransfected neurons in the same field of view $(n=4,5,4,3$, and $3[A P-1]$, and $4,3,3,5$ and 5 [AP-3] independent experiments for empty, AP-1a, AP-1b, AP-3a, and AP-3b vectors; all \pm SEM, ${ }^{* * *} p<0.001$ to empty vector, one-way ANOVA).

C

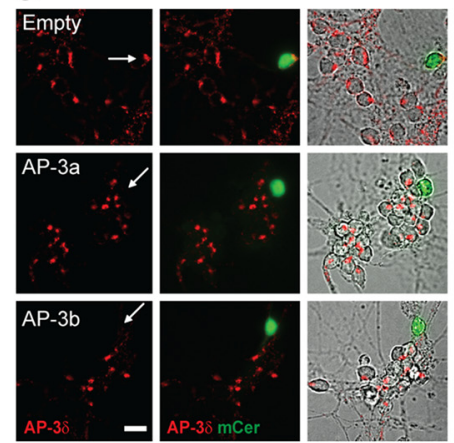

D

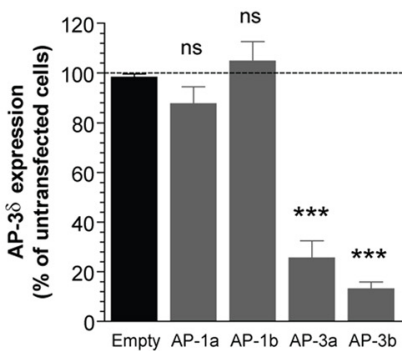


A

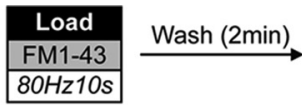

Immediate Unload

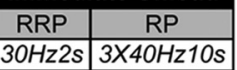

Rest (30 min)

B

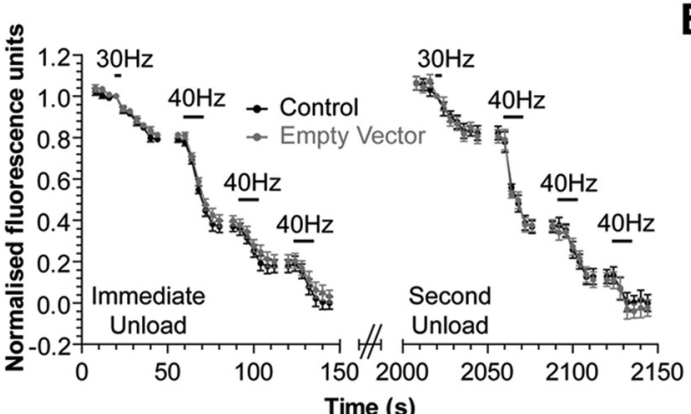

E

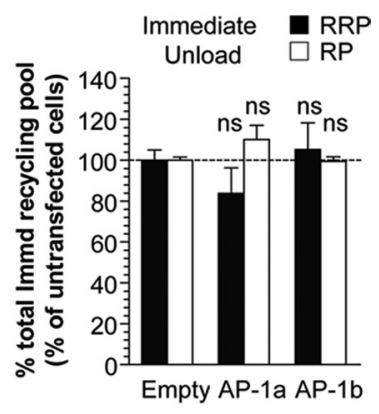

C

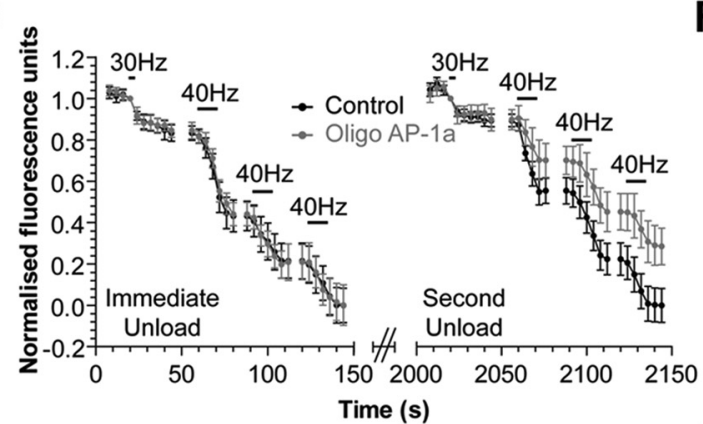

D

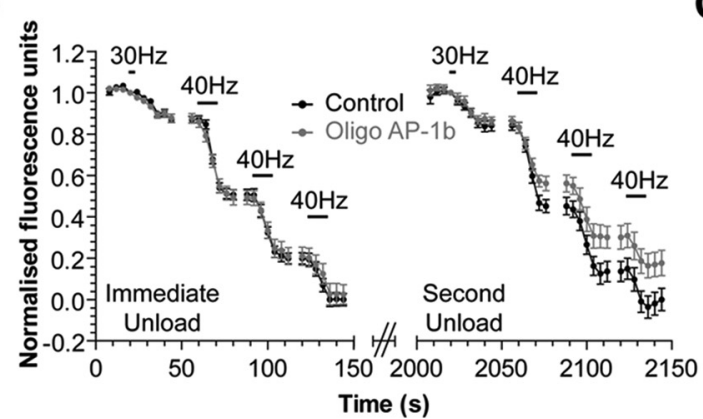

$\mathbf{F}$

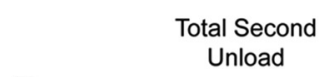

G

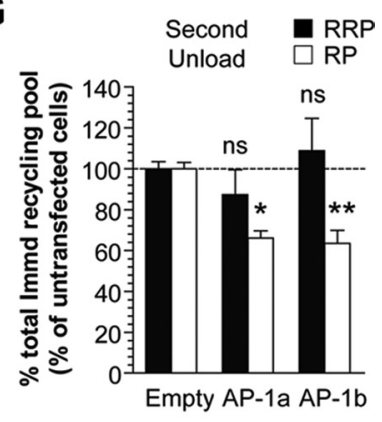

Figure 4. Reserve pool replenishment is inhibited by silencing AP-1 $\gamma$ expression. Cultures were transfected with shRNA that was either empty or expressing oligonucleotides against AP- $1 \gamma$ (AP-1a, AP-1b). A, After $72 \mathrm{~h}$, cultures were loaded with FM1-43 $(10 \mu \mathrm{M})$ using an 800 action potential $(80 \mathrm{~Hz})$ train. Dye was washed away immediately and after $2 \mathrm{~min}$ the RRP $(30 \mathrm{~Hz} 2 \mathrm{~s})$ and reserve pool (RP, 3 trains of $40 \mathrm{~Hz} 10 \mathrm{~s}$ ) were sequentially unloaded (Immediate Unload). The same unloading stimuli were delivered after a 30 min rest period (Second Unload). $\boldsymbol{B}-\boldsymbol{D}$, Representative traces of dye unloading during both the immediate and second unloads for cells transfected (gray) with either empty vector (B), AP-1a (C), or AP-1b (D). Graph also displays unloading from untransfected cells (control, black) from the same field of view. Both Immediate Unload and Second Unload traces are normalized to their respective recycling pool. $\boldsymbol{E}-\boldsymbol{G}$, Bar graphs display mean values of the size of the RRP and RP of the Immediate Unload (E), the Total Second Unload $(\boldsymbol{F})$, and the RRP and RP of the Second Unload (G). All values were first normalized to the total immediate recycling pool and then to the equivalent unload of untransfected nerve terminals in the same experiment (Empty, $n=$ 3; AP-1a, $n=3, \mathrm{AP}-1 \mathrm{~b}, n=4$ independent experiments; all $\pm \mathrm{SEM},{ }^{*} p<0.05,{ }^{* *} p<0.01,{ }^{* *} p<0.001$ to empty vector, one-way ANOVA).

10 min before the second unloading step $(\Delta \mathrm{S} 2)$ had no effect on the extent of FM2-10 unloading (SV exocytosis) when compared to control $(\Delta \mathrm{S} 2 / \Delta \mathrm{S} 1$ Control, $1.05 \pm 0.01 ; \mathrm{BFA}, 1.04 \pm 0.04 ; p=0.82$, Student's $t$ test). Thus, BFA does not directly interfere with SV exocytosis, confirming its effect was specific to reserve pool replenishment by bulk endosome-derived SVs.

Bulk endosome budding is impaired by silencing expression of either AP-1 or AP-3

BFA interferes with the ARF1-dependent recruitment of both AP-1 and AP-3 (Drake et al., 2000), implicating either or both AP complexes in the generation of SVs from bulk endosomes. To determine

their potential roles, we designed shRNA oligonucleotides specifically targeting the expression of either AP- $1 \gamma$ (oligonucleotides AP- $1 \mathrm{a}$ and $\mathrm{AP}-1 \mathrm{~b})$ or $\mathrm{AP}-3 \delta$ subunits (oligonucleotides AP-3a and AP-3b). These subunits were targeted since they are essential components of their respective AP complexes (Boehm and Bonifacino, 2001; Robinson, 2004). All oligonucleotides greatly reduced expression of their targeted subunit (Fig. 3). Importantly, no off-target effects on the reciprocal AP was observed (Fig. 3B,D).

The replenishment of the reserve SV pool provides an accurate readout of SV generation from bulk endosomes (Cheung et al., 2010); therefore, we used this assay to determine whether knockdown of either AP- $1 \gamma$ or AP- $3 \delta$ mimicked the effect of BFA (Fig. $2 D-F$ ). Cultures expressing shRNA against either subunit were loaded with FM1-43 using a train of 800 action potentials $(80 \mathrm{~Hz})$ followed by an immediate unload, rest period, and second unload in an identical experiment to before (Figs. $4 \mathrm{~A}$, $5 A$ ). The unloading of both the RRP and reserve pool in the immediate unloading step was unaffected by the silencing of either $\mathrm{AP}-1 \gamma$ or AP-3 $\delta$, suggesting no direct role for the AP-1 and AP-3 complexes in SV exocytosis (Figs. $4 B-E, 5 B-E$ ). However, after the 30 min rest period to allow SV generation, the total second unload of dye (reflecting the SV recycling pool) was significantly smaller in neurons where either AP- $1 \gamma$ or $\mathrm{AP}-3 \delta$ were silenced in comparison to empty vector (Figs. $4 F, 5 F$ ). When contributions from both RRP and reserve pool were determined, a selective inhibition of reserve pool unloading was observed for both $\mathrm{AP}-1 \gamma$ and $\mathrm{AP}-3 \delta$ silenced neurons (Fig. $4 B-E, G$ and $5 B-E, G$ ). Thus, a reduction in the expression of the essential subunits of either AP-1 complex or AP-3 complex resulted in a specific inhibition of reserve pool replenishment by SVs generated from bulk endosomes.

To confirm that the observed effects after either AP- $1 \gamma$ or AP- $3 \delta$ silencing were due to inhibition of SV generation from bulk endosomes, we monitored FM2-10 loading and unloading in shRNA-silenced cultures. This allows the effect of this maneuver to be determined on a population of SVs not generated by ADBE (Clayton et al., 2009). Dye was loaded into cultures using a train of 800 action potentials $(80 \mathrm{~Hz})$, and the extent and kinetics of SV exocytosis were monitored using a subsequent unloading stimulus of 800 action potentials $(40 \mathrm{~Hz}$, Fig. 6 A). The kinetics of dye unloading in all neurons transfected with silencing shRNAs were indistinguishable from those transfected with empty shRNA vectors, confirming no direct effect of AP- $1 \gamma$ and AP-3 $\delta$ silencing on SV exocytosis (Fig. 6B-E). Furthermore, there was no difference in the extent of dye unloading (Fig. $6 F-I$ ), which re- 
A

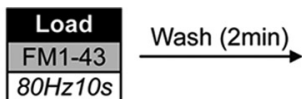

Immediate Unload

\begin{tabular}{|c|c|}
\hline RRP & RP \\
\hline $30 H z 2 s$ & $3 X 40 H z 10 s$ \\
\hline
\end{tabular}

$\longrightarrow$

Rest (30 min)

B

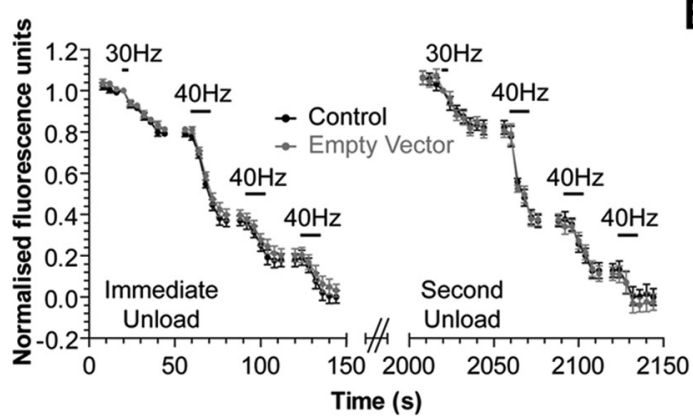

C

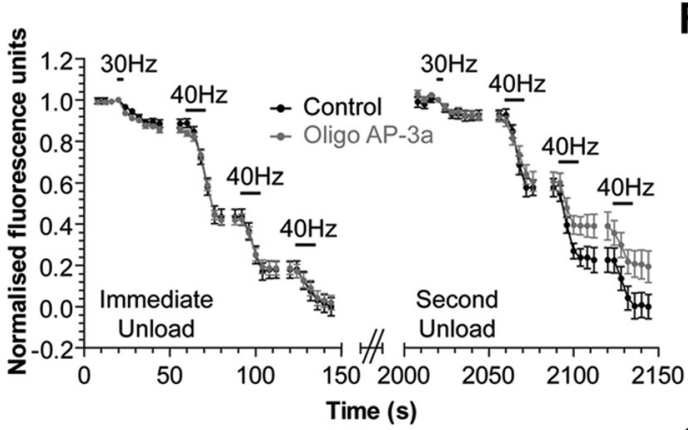

D

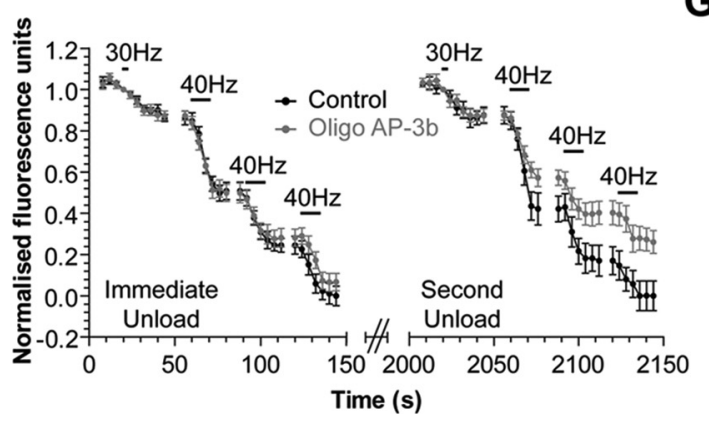

G
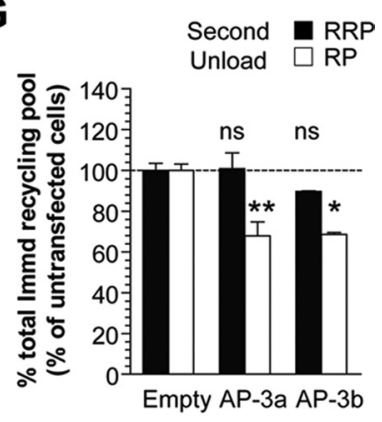

Figure 5. Reserve pool replenishment is inhibited by silencing AP- $3 \delta$ expression. Cultures were transfected with shRNA that was either empty or expressing oligonucleotides against AP-3 $\delta$ (AP-3a, AP-3b). $A$, After $72 \mathrm{~h}$, cultures were loaded with FM1-43 $(10 \mu \mathrm{M})$ using an 800 action potential $(80 \mathrm{~Hz})$ train. Dye was washed away immediately and after $2 \mathrm{~min}$ the RRP $(30 \mathrm{~Hz}, 2 \mathrm{~s})$ and reserve pool ( $\mathrm{RP}$, three trains of $40 \mathrm{~Hz}, 10 \mathrm{~s}$ ) were sequentially unloaded (Immediate Unload). The same unloading stimuli were delivered after a $30 \mathrm{~min}$ rest period (Second Unload). $\boldsymbol{B}-\boldsymbol{D}$, Representative traces of dye unloading during both the immediate and second unloads for cells transfected (gray) with either empty vector (B), AP-3a (C), or AP-3b (D). Graph also displays unloading from untransfected cells (Control, black) from the same field of view. Both Immediate Unload and Second Unload traces are normalized to their respective recycling pool. $\boldsymbol{E}-\boldsymbol{G}$, Bar graphs display mean values of the size of the RRP and RP of the Immediate Unload $(\boldsymbol{E})$, the Total Second Unload $(\boldsymbol{F})$, and the RRP and RP of the Second Unload (G). All values were first normalized to the total immediate recycling pool and then to the equivalent unload of untransfected nerve terminals in the same experiment (Empty, $n=$ $3 ; \mathrm{AP}-3 \mathrm{a}, n=6, \mathrm{AP}-3 \mathrm{~b}, n=3$ independent experiments; all $\pm \mathrm{SEM},{ }^{*} p<0.05,{ }^{* *} p<0.01$, to empty vector, one-way ANOVA).

flects replenishment of the SV recycling pool by CME-derived SVs (Cheung et al., 2010). Thus, silencing the expression of either AP- $1 \gamma$ or AP- $3 \delta$ had no effect on the retrieval, recycling, or exocytosis of CME-generated SVs (Fig. 6E,I), confirming their specific role in ADBE.

The arrest of SV budding and subsequent reserve pool replenishment caused by the knockdown of either AP- $1 \gamma$ or AP- $3 \delta$ may have resulted from an upstream inhibition in the generation of bulk endosomes. To assess this, we monitored the uptake of high molecular weight fluorescent dextrans (tetramethylrhodamine-dextran, 40 $\mathrm{kDa}$ ), which are too large to be accumulated by CME (Clayton et al., 2008; Clayton and Cousin, 2009b). Cultures transfected with shRNA against either AP- $1 \gamma$ or AP- $3 \delta$ were stimulated with a train of 800 action potentials $(80 \mathrm{~Hz})$ in the presence of dextran to evoke its internalization by ADBE. In all instances, dextran uptake was comparable to uptake in neurons transfected with empty shRNA vectors (Fig. 7). Thus, the arrest in reserve pool replenishment observed in AP- $1 \gamma$ - or AP- $3 \delta$-silenced neurons was due to defective SV generation from bulk endosomes and not the faulty formation of bulk endosomes.

\section{$\mathrm{AP}-1$ and AP-3 generate the same SV population from bulk endosomes} We have shown that the AP-1 and AP-3 complexes are individually required for SV generation from bulk endosomes. There are two possible explanations for this requirement. These protein complexes could either be producing two separate populations of SVs in parallel budding pathways, or be sequentially required in the same budding pathway. To determine this, we simultaneously silenced the expression of AP- $1 \gamma$ and AP- $3 \delta$ in the same neurons. If AP-1 and AP-3 had essential roles in parallel pathways, this would be visualized as an additive inhibition of reserve pool replenishment, whereas generation of the same population of SVs would produce no additional inhibition.

Cultures were loaded with FM1-43 using 800 action potentials $(80 \mathrm{~Hz})$ followed by an immediate unload, rest period, and second unload as before (Fig. 8A). Both RRP and reserve pool unloading during immediate unloading were unaffected by the simultaneous silencing of both AP- $1 \gamma$ and AP- $3 \delta$ (Fig. $8 C, D$ ). Similarly, RRP replenishment after 30 min was unaffected (Fig. $8 E$ ). Importantly, the inhibition of reserve pool replenishment in the simultaneous absence of both AP- $1 \gamma$ and AP- $3 \delta$ was not significantly different to the inhibition observed by their single knockdown (Fig. $8 E$, compared to Figs. $4 \mathrm{G}$ and $5 \mathrm{G}$, reserve pool size as percentage of empty vector; shRNA AP-1b, $68.6 \pm 1.0$; AP-3b, $63.5 \pm 6.4$; AP- $1 \mathrm{~b}$ plus AP-3b, $61.7 \pm 9.7$; not significant one-way ANOVA). In addition, silencing of either AP- $1 \gamma$ or AP- $3 \delta$ expression did not produce any additional inhibition of reserve pool replenishment to that already observed with BFA (Fig. 9). Since the knockdown of AP- $1 \gamma$ and AP- $3 \delta$ has no additive effect on reserve pool replenishment, we conclude that they control the same SV budding pathway and thus generate the same SV population from bulk endosomes.

\section{Discussion}

We have shown an essential requirement for both AP-1 and AP-3 complexes in the generation of SVs from bulk endosomes, which translates into a specific replenishment of the reserve SV pool. 

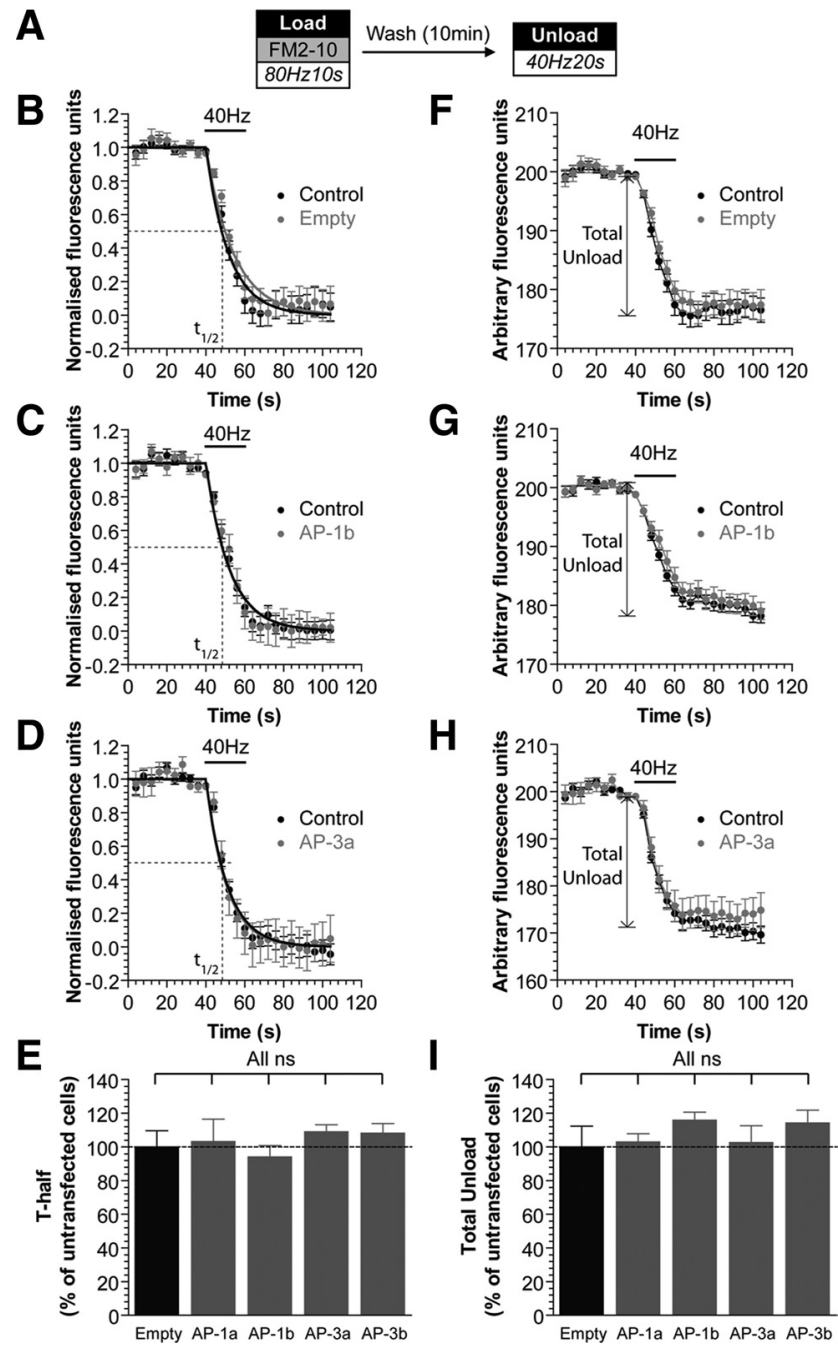

A

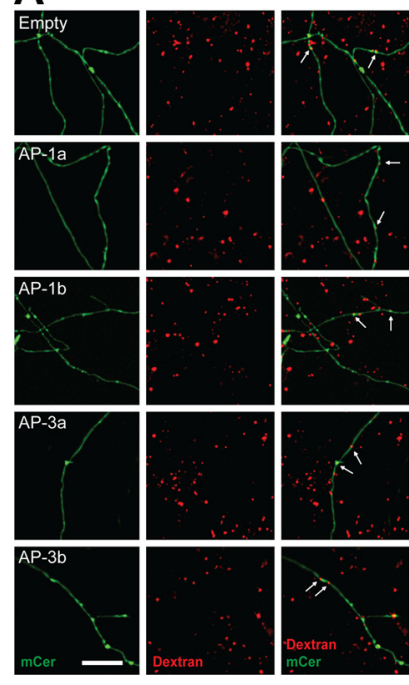

B

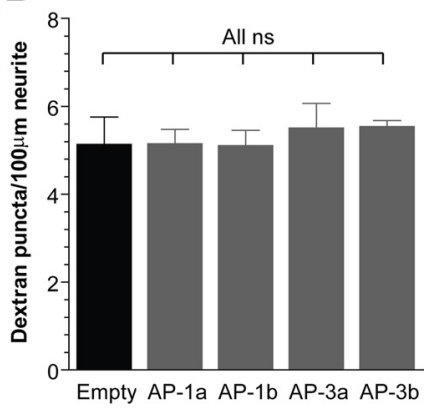

Figure 7. Silencing AP-1 $\gamma$ or AP-3 $\delta$ expression does not inhibit ADBE. Cultures were transfected with shRNA that was either empty or expressing oligonucleotides against either AP-1 $\gamma$ (AP-1a, AP-1b) or AP-3 $\delta$ (AP-3a, AP-3b). After $72 \mathrm{~h}$, cultures were stimulated with a train of 800 action potentials $(80 \mathrm{~Hz})$ in the presence of $40 \mathrm{kDa}$ tetramethylrhodamine-dextran $(50 \mu \mathrm{m})$. Dextran was washed away immediately after stimulation. $\boldsymbol{A}$, Representative images of dextran loading are shown. Left panels show neurons transfected with the shRNA vector (green). Middle panels show dextran uptake (red). Right panels show a merged image of transfected neuron (green) and dextran uptake (red). White arrows indicate dextran puncta on transfected neurites (scale bar, $10 \mu \mathrm{m}$ ). B, Bar graph displaying the mean number of dextran puncta per $100 \mu \mathrm{m}$ of neurite (Empty, $n=3 ; \mathrm{AP}-1 \mathrm{a}, n=4 ; \mathrm{AP}-1 \mathrm{~b}, n=3 ; \mathrm{AP}-3 \mathrm{a}, n=3 ; \mathrm{AP}-3 \mathrm{~b}, n=3$ independent experiments; all \pm SEM, one-way ANOVA).

neurons have shown that AP-3 is required in concert with the BLOC-1 complex for the traffic of SV protein cargo from cell bodies to nerve terminals (Larimore et al., 2011). The presynaptic localization of both AP-1 and AP-3 complexes (Glyvuk et al., 2010; Newell-Litwa et al., 2010), coupled to the fact that BFA only affects $\mathrm{SV}$ recycling during elevated neuronal activity (Voglmaier et al., 2006; Kim and Ryan, 2009), suggested that either or both could be required for SV generation from bulk endosomes. To determine this, we designed assays to specifically monitor SV generation from bulk endosomes as opposed to classical early endosomes or other endosomal compartments. The strategy involved loading bulk endosomes with a reporter molecule (FM1-43 or HRP), depleting nerve terminals of SVs that were generated by CME, and then following the generation of new SVs from bulk endosomes (Cheung et al., 2010). This approach permits a pharmacological intervention (such as BFA application) once ADBE is complete, allowing separation of effects on bulk endosome budding from any potential effects on plasma membrane endocytosis. It also allows a molecular dissection of the replenishment of specific SV pools by separate SV endocytosis modes via use of either overexpression or shRNA silencing vectors, providing these maneuvers have no direct effects on SV exocytosis (Cheung et al., 2010).

A measurable reserve pool of SVs can be generated even after pharmacological or molecular ablation of AP-1 and AP-3 function, which raises questions regarding the origin of these SVs. The most likely scenario is that a few SVs are still being generated from bulk endosomes due to incomplete knockdown of AP-1 and AP-3 or incomplete inhibition by BFA. Another potential possibility is that a different adaptor protein may substitute for AP-1 or AP-3, as seen when AP-2 expression is silenced during CME (Kim and Ryan, 2009). It is unlikely that these remaining reserve

shown clear evidence that the AP-3 complex is essential for vesicle 1998; Blumstein et al., 2001). Furthermore, studies in central

Evidence for this is as follows: (1) BFA inhibits generation of SVs from HRP-labeled bulk endosomes; (2) BFA and shRNA knockdown of either AP-1 or AP-3 inhibits the replishment of the reserve pool from bulk endosomes; and (3) AP-1 and AP-3 play no role at the plasma membrane in either $\mathrm{ADBE}$ or $\mathrm{CME}$.

Previous biochemical studies in neuroendocrine cells have shown clear evidence that the AP-3 complex is essential for vesicle 
A

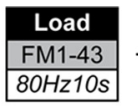

$\stackrel{\text { Wash (2min) }}{\longrightarrow}$

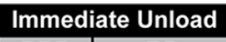

RRP

$\stackrel{\text { Rest (30 min) }}{\longrightarrow}$

Second Unload

\begin{tabular}{|c|c|}
\hline RRP & RP \\
\hline $30 H z 2 s$ & $3 \times 40 H z 10 s$ \\
\hline
\end{tabular}

B

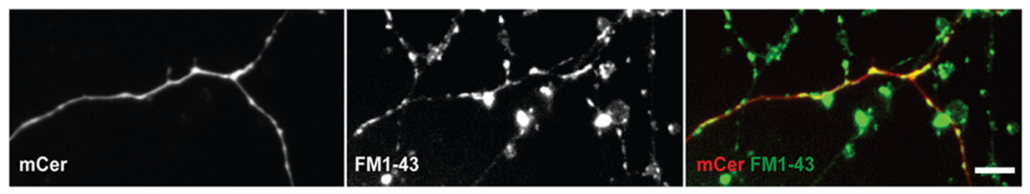

C

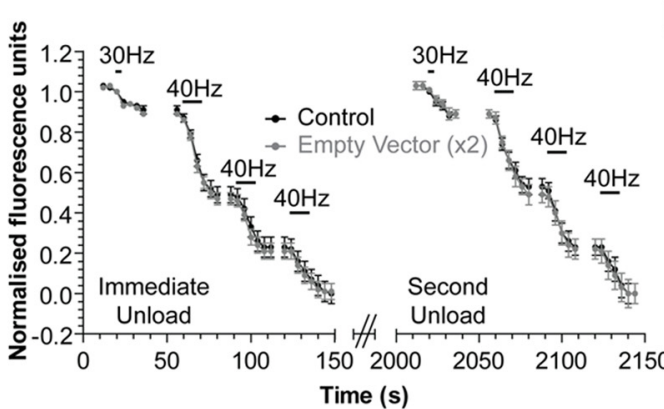

D
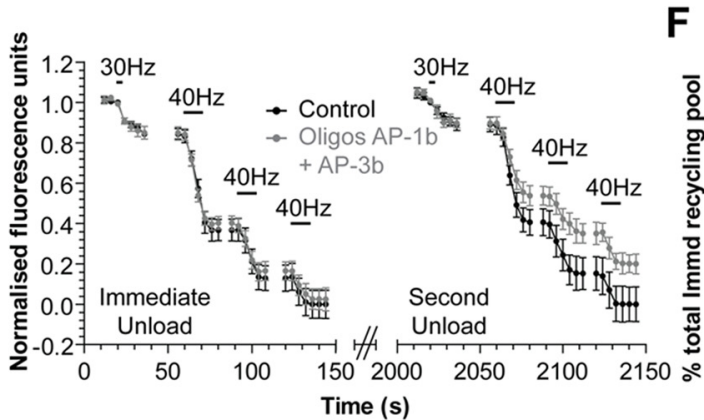

E
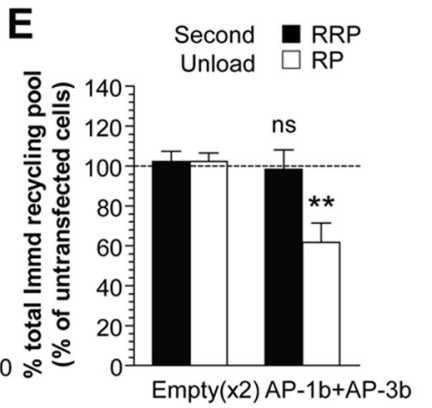

$\mathrm{F}$

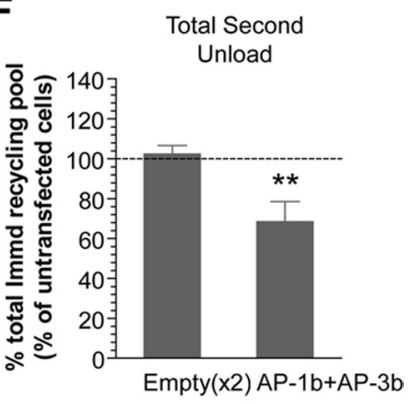

Figure 8. Simultaneous silencing of AP- $1 \gamma$ or $A P-3 \delta$ inhibits reserve pool replenishment to the same extent as individual silencing. Cultures were cotransfected with either shRNA against AP- $1 \gamma$ and AP-3 $\delta(A P-1 b, A P-3 b)$ or a double concentration of empty shRNA vector. $A$, After $72 \mathrm{~h}$, cultures were loaded with FM1-43 $(10 \mu \mathrm{m})$ using an 800 action potential $(80 \mathrm{~Hz})$ train. Dye was washed away immediately and after $2 \mathrm{~min}$ the $\operatorname{RRP}(30 \mathrm{~Hz}, 2 \mathrm{~s})$ and reserve pool (RP, three trains of $40 \mathrm{~Hz}, 10 \mathrm{~s})$ were sequentially unloaded (Immediate Unload). The same unloading stimuli were delivered after a $30 \mathrm{~min}$ rest period (Second Unload). B, Representative images are shown for neurons cotransfected with AP-1b and AP-3b shRNA vector (left, mCer, mCerulean), FM1-43 loading in the same field of view (middle, FM1-43), and a merged image of transfected neuron (red) and FM1-43 loading (green). Scale bar, $10 \mu \mathrm{m}$. C, D Representative traces of dye unloading during both the immediate and second unloads for cells transfected (gray) with either a double concentration of empty vector $(\boldsymbol{C})$ or cotransfection with AP-1b and AP-3b (D). Graphs also display unloading from untransfected cells (control, black) from the same field of view. Both Immediate Unload and Second Unload traces are normalized to the total recycling pool. $\boldsymbol{E}, \boldsymbol{F}$, Bar graphs display mean values of the size of the RRP and RP of the Second Unload $(\boldsymbol{E})$ and the Total Second Unload $(\boldsymbol{F})$. All values were first normalized to the total immediate recycling pool and then to the equivalent unload of untransfected nerve terminals in the same experiment $\left(n=3\right.$ independent experiments; all \pm SEM, ${ }^{* *} p<$ 0.01 , one-way ANOVA and Student's $t$ test for $\boldsymbol{E}$ and $\boldsymbol{F}$ respectively).

pool SVs are generated at the plasma membrane by CME, since reserve pool replenishment can be ablated by addition of the dynamin antagonist dynasore after dye loading is complete (G. Cheung, unpublished observations). Thus, the SVs that replenish the reserve pool are generated by a dynamin-dependent event that occurs after stimulation is complete, suggesting they are derived from bulk endosomes.

\section{Different AP complexes generate SVs from \\ different compartments}

Different APs concentrate protein cargo during vesicle budding at different membrane compartments (Robinson, 2004). For example, AP-1 has a defined role in budding vesicles from the transGolgi network (Ghosh et al., 2003). In addition to this classical role, AP-1 has been implicated in vesicle budding from recycling endosomes to either the cell surface or the Golgi complex (Pagano et al., 2004), whereas AP-3 is present on early endosomes and aids delivery of cargo to late endosomes and lysosomes (Newell-Litwa et al., 2007). AP-3 is also essential for the generation of vesicles from endosomes in neuroendocrine cells and for sorting SV cargo for transport to nerve terminals (Blumstein et al., 2001; Larimore et al., 2011). In contrast, $\mathrm{AP}-2$ is required for $\mathrm{CME}$ at the plasma membrane (Robinson, 2004). Recent studies using shRNA silencing of AP-2 in primary hippocampal cultures confirmed that it plays a role in SV endocytosis during mild stimulation, whereas BFA treatment or knockdown of AP-1 $\gamma$ had no effect (Kim and Ryan, 2009). Our studies agree with these findings, showing that retrieval of SVs by CME or dextran internalization by ADBE was not affected by knockdown of either AP- $1 \gamma$ or AP- $3 \delta$, highlighting the lack of a role for either AP- 1 or AP-3 at the nerve terminal plasma membrane.

We have shown an essential role for both AP-1 and AP-3 in the generation of SVs from bulk endosomes. This key shared molecular function explains previous observations in a number of neuronal systems. For example, BFA inhibited a slow route of SV protein cargo traffic during high intensity, but not low intensity, stimulation in primary hippocampal neurons (Voglmaier et al., 2006). Also, deletion of the AP- $3 \mu \mathrm{B}$ subunit reduced SV numbers in inhibitory nerve terminals (Nakatsu et al., 2004), which typically have higher activity rates (Bartos et al., 2007). Mocha mice (which are null for AP- $3 \delta$ and have little or no other AP-3 subunits) have SVs of irregular size (Newell-Litwa et al., 2010). They also display defects in asynchronous neurotransmitter release and a higher mEPSC frequency (Scheuber et al., 2006). Mice with a genetic ablation of AP- $1 \sigma \mathrm{B}$ have a marked reduction of SVs, a phenotype that is exacerbated by stimulation (Glyvuk et al., 2010). Finally, cultured neurons from AP-1 $\sigma \mathrm{B}$-null mice displayed a stimulation-dependent increase in endosome number compared to wild-type and a robust defect in the replenishment of the recycling pool (Glyvuk et al., 2010). An arrest of SV generation from bulk endosomes specifically during ADBE explains these prior observations and provides a direct molecular locus for these AP complexes in the SV life cycle.

\section{Dual essential requirement for AP-1 and AP-3 in SV generation from bulk endosomes}

We demonstrate a shared requirement for both AP-1 and AP-3 in SV reserve pool replenishment from bulk endosomes. This suggests that: (1) AP-1 and AP-3 are not functionally redundant; and (2) they both have key and specific roles in the generation of SVs from bulk endosomes. In different cellular systems AP-1 and AP-3 have displayed varying degrees of functional overlap. For example, they have partially redundant functions in sorting cargo 


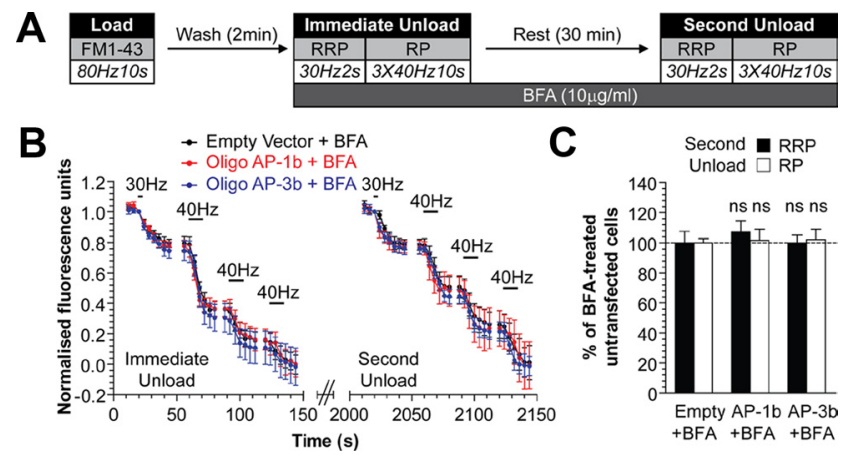

Figure 9. Silencing of either AP-1 $\gamma$ or AP- $3 \delta$ does not increase the inhibition of reserve pool replenishment observed with $B F A$. Cultures were transfected with shRNA that was either empty or expressing oligonucleotides against AP- $1 \gamma$ (AP-1b) or AP- $3 \delta$ (AP-3b). $A$, After $72 \mathrm{~h}$, cultures were loaded with FM1-43 $(10 \mu \mathrm{M})$ using an 800 action potential $(80 \mathrm{~Hz})$ train. Dye was washed away immediately and after 2 min the $R R P(30 \mathrm{~Hz}, 2 \mathrm{~s})$ and reserve pool (RP, 3 trains of $40 \mathrm{~Hz}$, 10 s) were sequentially unloaded (Immediate Unload). The same unloading stimuli were delivered after a 30 min rest period (Second Unload). Brefeldin A $(10 \mu \mathrm{g} / \mathrm{ml}$ ) was exposed to cultures before the Immediate Unload and was present thereafter. $\boldsymbol{B}$, Representative traces of dye unloading during both the immediate and second unloads for cells transfected with either empty vector plus BFA (black), AP-1b plus BFA (red), or AP-3b plus BFA (blue). Both Immediate Unload and Second Unload traces are normalized to their respective recycling pool. C, Bar graphs display mean values of the size of the RRP and RP of the second unload. All values were first normalized to the total immediate recycling pool and then to the equivalent unload of untransfected nerve terminals in the same experiment ( $n=3$ independent experiments; all \pm SEM, one-way ANOVA).

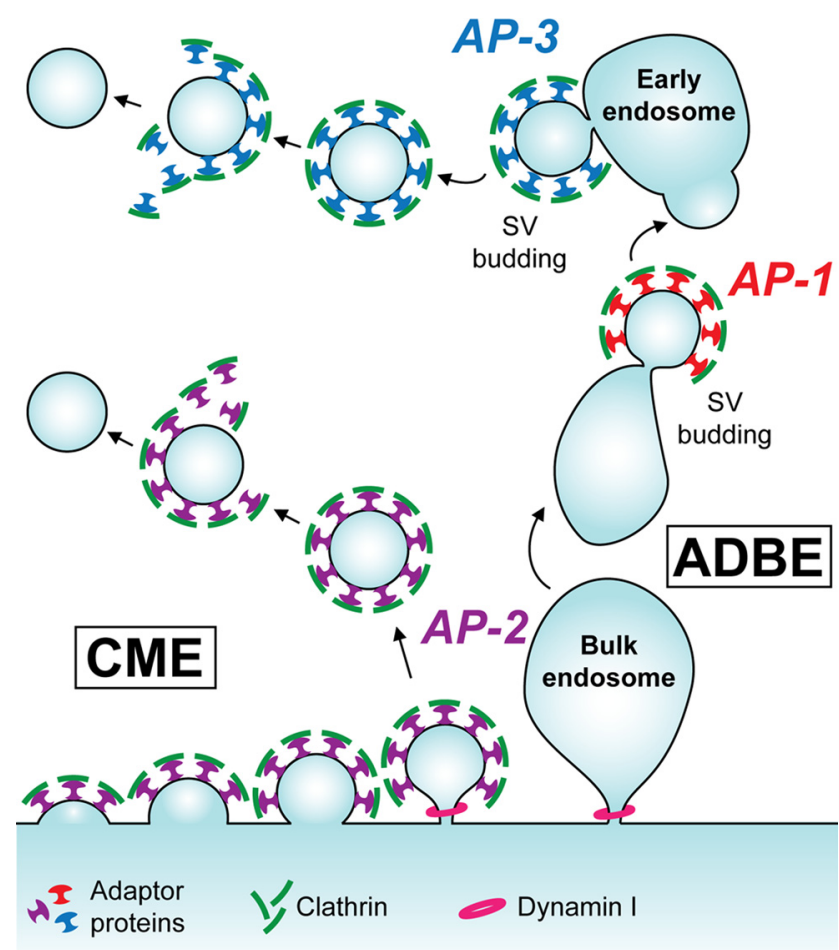

Figure 10. Potential shared roles for AP-1 and AP-3 complexes in ADBE. The dual requirement for AP- 1 and AP-3 in ADBE can be explained by a sequential requirement for AP- 1 and AP-3. After formation of the bulk endosome, an immature SV is formed in an AP-1-dependent manner. This immature SV delivers SV cargo to a classical early endosome, and then a mature SV (with a full complement of cargo) buds from this compartment using AP-3.

to melanosomes (Theos et al., 2005; Delevoye et al., 2009; Lakkaraju et al., 2009), where AP-1 and AP-3 form distinct vesicles with different melanosome cargo in in vitro budding assays (Chapuy et al., 2008). However, this does not seem to occur at the bulk endosome, since we have shown that both AP complexes are essential for the generation of the same SV pool. This is not due to neurons exclusively expressing either AP-1 or AP-3, since we have shown by immunofluorescence that almost all neurons in culture express both AP- $1 \gamma$ and AP- $3 \delta(92.3 \pm 2.2 \%$ AP- $-1 \gamma$; $88.1 \pm 1.4 \% \mathrm{AP}-3 \delta$ ).

Why are AP-1 and AP-3 both essential for the production of SVs from bulk endosomes? One possibility is that these complexes work in a sequential manner to generate SVs. In this model a precursor SV buds from a bulk endosome (using for example AP-1) and fuses with a classical early sorting endosome. A mature SV may then bud from the early endosome (using AP-3) and replenish the reserve pool (Fig. 10). There is no current morphological evidence to suggest that this occurs; however, recent work from a number of laboratories have suggested that movement of SV membrane through classical early endosomes may contribute toward SV recycling in central nerve terminals (Hoopmann et al., 2010; Uytterhoeven et al., 2011). We are currently testing this hypothesis by examining the potential contributions from classical early endosomes to SV recycling during different stimulation intensities.

The shared essential roles for both AP-1 and AP-3 in SV budding from bulk endosomes highlight a key conceptual divergence between ADBE and CME. During CME, SV protein cargo is concentrated by AP- 2 and potentially other non-classical adaptors at the plasma membrane (Jung and Haucke, 2007; Kelly and Owen, 2011). However, the essential requirement for AP- 1 and AP-3 at bulk endosomes suggests that little or no sorting of SV protein cargo occurs at the plasma membrane during ADBE. This makes conceptual sense, since the major physiological role of ADBE is to rapidly correct for large changes in plasma membrane surface area, rather than create a fully functional SV. Other forms of fluid phase uptake actively exclude specific plasma membrane proteins (Mercanti et al., 2006), suggesting that ADBE may use a similar mechanism, allowing the rate-limiting step of SV cargo clustering to be performed once inside the neuron. Thus, during ADBE, SV cargo selection and processing may occur at the bulk endosome rather than at the plasma membrane.

In summary, we have demonstrated a molecular locus of action for AP-1 and AP-3 in the SV life cycle in central nerve terminals. This essential molecular requirement for SV generation from bulk endosomes will provide a novel point of intervention to determine how ADBE controls neurotransmission in both neuronal physiology and pathology.

\section{References}

Bartos M, Vida I, Jonas P (2007) Synaptic mechanisms of synchronized gamma oscillations in inhibitory interneuron networks. Nat Rev Neurosci 8:45-56.

Blumstein J, Faundez V, Nakatsu F, Saito T, Ohno H, Kelly RB (2001) The neuronal form of adaptor protein-3 is required for synaptic vesicle formation from endosomes. J Neurosci 21:8034-8042.

Boehm M, Bonifacino JS (2001) Adaptins. The final recount. Mol Biol Cell 12:2907-2920.

Chapuy B, Tikkanen R, Mühlhausen C, Wenzel D, von Figura K, Höning S (2008) AP-1 and AP-3 mediate sorting of melanosomal and lysosomal membrane proteins into distinct post-Golgi trafficking pathways. Traffic 9:1157-1172.

Cheung G, Jupp OJ, Cousin MA (2010) Activity-dependent bulk endocytosis and clathrin-dependent endocytosis replenish specific synaptic vesicle pools in central nerve terminals. J Neurosci 30:8151-8161.

Clayton EL, Cousin MA (2008) Differential labelling of bulk endocytosis in nerve terminals by FM dyes. Neurochem Int 53:51-55.

Clayton EL, Cousin MA (2009a) The molecular physiology of activitydependent bulk endocytosis of synaptic vesicles. J Neurochem 111:901-914. 
Clayton EL, Cousin MA (2009b) Quantitative monitoring of activitydependent bulk endocytosis of synaptic vesicle membrane by fluorescent dextran imaging. J Neurosci Methods 185:76-81.

Clayton EL, Evans GJ, Cousin MA (2008) Bulk synaptic vesicle endocytosis is rapidly triggered during strong stimulation. J Neurosci 28:6627-6632.

Clayton EL, Anggono V, Smillie KJ, Chau N, Robinson PJ, Cousin MA (2009) The phospho-dependent dynamin-syndapin interaction triggers activity-dependent bulk endocytosis of synaptic vesicles. J Neurosci 29:7706-7717.

Delevoye C, Hurbain I, Tenza D, Sibarita JB, Uzan-Gafsou S, Ohno H, Geerts WJ, Verkleij AJ, Salamero J, Marks MS, Raposo G (2009) AP-1 and KIF13A coordinate endosomal sorting and positioning during melanosome biogenesis. J Cell Biol 187:247-264.

Drake MT, Zhu Y, Kornfeld S (2000) The assembly of AP-3 adaptor complex-containing clathrin-coated vesicles on synthetic liposomes. Mol Biol Cell 11:3723-3736.

Faúndez V, Horng JT, Kelly RB (1998) A function for the AP3 coat complex in synaptic vesicle formation from endosomes. Cell 93:423-432.

Ghosh P, Dahms NM, Kornfeld S (2003) Mannose 6-phosphate receptors: new twists in the tale. Nat Rev Mol Cell Biol 4:202-212.

Glyvuk N, Tsytsyura Y, Geumann C, D’Hooge R, Hüve J, Kratzke M, Baltes J, Böning D, Klingauf J, Schu P (2010) AP-1/sigma1B-adaptin mediates endosomal synaptic vesicle recycling, learning and memory. EMBO J 29:1318-1330.

Granseth B, Odermatt B, Royle SJ, Lagnado L (2006) Clathrin-mediated endocytosis is the dominant mechanism of vesicle retrieval at hippocampal synapses. Neuron 51:773-786.

Heerssen H, Fetter RD, Davis GW (2008) Clathrin dependence of synapticvesicle formation at the Drosophila neuromuscular junction. Curr Biol 18:401-409.

Hirst J, Barlow LD, Francisco GC, Sahlender DA, Seaman MN, Dacks JB, Robinson MS (2011) The fifth adaptor protein complex. PLoS Biol 9:e1001170.

Hoopmann P, Punge A, Barysch SV, Westphal V, Bückers J, Opazo F, Bethani I, Lauterbach MA, Hell SW, Rizzoli SO (2010) Endosomal sorting of readily releasable synaptic vesicles. Proc Natl Acad Sci U S A 107:19055-19060.

Jung N, Haucke V (2007) Clathrin-mediated endocytosis at synapses. Traffic 8:1129-1136.

Kasprowicz J, Kuenen S, Miskiewicz K, Habets RL, Smitz L, Verstreken P (2008) Inactivation of clathrin heavy chain inhibits synaptic recycling but allows bulk membrane uptake. J Cell Biol 182:1007-1016.

Kelly BT, Owen DJ (2011) Endocytic sorting of transmembrane protein cargo. Curr Opin Cell Biol 23:404-412.

Kim SH, Ryan TA (2009) Synaptic vesicle recycling at CNS snapses without AP-2. J Neurosci 29:3865-3874.

Lakkaraju A, Carvajal-Gonzalez JM, Rodriguez-Boulan E (2009) It takes two to tango to the melanosome. J Cell Biol 187:161-163.

Larimore J, Tornieri K, Ryder PV, Gokhale A, Zlatic SA, Craige B, Lee JD, Talbot K, Pare JF, Smith Y, Faundez V (2011) The schizophrenia susceptibility factor dysbindin and its associated complex sort cargoes from cell bodies to the synapse. Mol Biol Cell 22:4854-4867.

Mercanti V, Charette SJ, Bennett N, Ryckewaert JJ, Letourneur F, Cosson
P (2006) Selective membrane exclusion in phagocytic and macropinocytic cups. J Cell Sci 119:4079-4087.

Nakatsu F, Okada M, Mori F, Kumazawa N, Iwasa H, Zhu G, Kasagi Y, Kamiya H, Harada A, Nishimura K, Takeuchi A, Miyazaki T, Watanabe M, Yuasa S, Manabe T, Wakabayashi K, Kaneko S, Saito T, Ohno H (2004) Defective function of GABA-containing synaptic vesicles in mice lacking the AP-3B clathrin adaptor. J Cell Biol 167:293-302.

Newell-Litwa K, Seong E, Burmeister M, Faundez V (2007) Neuronal and non-neuronal functions of the AP-3 sorting machinery. J Cell Sci 120:531-541.

Newell-Litwa K, Chintala S, Jenkins S, Pare JF, McGaha L, Smith Y, Faundez V (2010) Hermansky-Pudlak protein complexes, AP-3 and BLOC-1, differentially regulate presynaptic composition in the striatum and hippocampus. J Neurosci 30:820-831.

Pagano A, Crottet P, Prescianotto-Baschong C, Spiess M (2004) In vitro formation of recycling vesicles from endosomes requires adaptor protein$1 /$ clathrin and is regulated by rab4 and the connector rabaptin-5. Mol Biol Cell 15:4990-5000.

Richards DA, Guatimosim C, Betz WJ (2000) Two endocytic recycling routes selectively fill two vesicle pools in frog motor nerve terminals. Neuron 27:551-559.

Robinson MS (2004) Adaptable adaptors for coated vesicles. Trends Cell Biol 14:167-174.

Royle SJ, Lagnado L (2003) Endocytosis at the synaptic terminal. J Physiol 553:345-355.

Scheuber A, Rudge R, Danglot L, Raposo G, Binz T, Poncer JC, Galli T (2006) Loss of AP-3 function affects spontaneous and evoked release at hippocampal mossy fiber synapses. Proc Natl Acad Sci U S A 103:16562-16567.

Shupliakov O (2009) The synaptic vesicle cluster: a source of endocytic proteins during neurotransmitter release. Neuroscience 158:204-210.

Südhof TC (2000) The synaptic vesicle cycle revisited. Neuron 28:317-320.

Takamori S, Holt M, Stenius K, Lemke EA, Grønborg M, Riedel D, Urlaub H, Schenck S, Brügger B, Ringler P, Müller SA, Rammner B, Gräter F, Hub JS, De Groot BL, Mieskes G, Moriyama Y, Klingauf J, Grubmüller H, Heuser J, Wieland F, Jahn R (2006) Molecular anatomy of a trafficking organelle. Cell 127:831-846.

Tan TC, Valova VA, Malladi CS, Graham ME, Berven LA, Jupp OJ, Hansra G, McClure SJ, Sarcevic B, Boadle RA, Larsen MR, Cousin MA, Robinson PJ (2003) Cdk5 is essential for synaptic vesicle endocytosis. Nat Cell Biol 5:701-710.

Theos AC, Tenza D, Martina JA, Hurbain I, Peden AA, Sviderskaya EV, Stewart A, Robinson MS, Bennett DC, Cutler DF, Bonifacino JS, Marks MS, Raposo G (2005) Functions of adaptor protein (AP)-3 and AP-1 in tyrosinase sorting from endosomes to melanosomes. Mol Biol Cell 16:5356-5372.

Uytterhoeven V, Kuenen S, Kasprowicz J, Miskiewicz K, Verstreken P (2011) Loss of skywalker reveals synaptic endosomes as sorting stations for synaptic vesicle proteins. Cell 145:117-132.

Voglmaier SM, Kam K, Yang H, Fortin DL, Hua Z, Nicoll RA, Edwards RH (2006) Distinct endocytic pathways control the rate and extent of synaptic vesicle protein recycling. Neuron 51:71-84.

Zhu Y, Xu J, Heinemann SF (2009) Two pathways of synaptic vesicle retrieval revealed by single-vesicle imaging. Neuron 61:397-411. 